\title{
Esencia y trascendencia de la obra de Jean Piaget (1896-1980)
}

\author{
Silvia Parrat-Dayan \\ Archives Jean Piaget \\ Universidad de Ginebra
}

Recibido: 15 de marzo de 2012 / Aprobado: 20 de abril de 2012

Piaget nos ha dejado una impresionante obra escrita que revolucionó la epistemología y la psicología de su época. Su punto de vista constituye una observación coherente sobre cómo estudiar y entender el conocimiento. A diferencia de otros epistemólogos, el interés de Piaget no reside en estudiar la naturaleza del conocimiento, sino en preguntarse cómo se pasa de un estado de conocimiento menos potente a un estado de conocimiento más potente.

Piaget siempre se interesó en saber cuáles son los mecanismos que permiten la emergencia de "lo nuevo". Y trata estas preguntas en tanto que psicólogo, lo que muestra la originalidad de su programa. La teoría de Piaget es innovadora por muchos aspectos. Señalemos entre otros la naturaleza interdisciplinaria de su trabajo, los problemas que plantea, las estrategias utilizadas al plantearlos y la manera de resolverlos.

psicología genética / epistemología / constructivismo / Piaget

\section{Essence and transcendence of the work of Jean Piaget (1896-1980)}

Piaget has endowed us with an enormous amount of written work that in his time revolutionized epistemology and psychology. His point of view is a coherent observation on how to study and understand knowledge. In contrast to other epistemologists, Piaget did not aimed to studying the nature of knowledge but to ask how is that we change from a state of knowledge less powerful to a more powerful. Piaget was always interested in determining the mechanisms that allow the emergence of "the novel". And these questions are posed from the point of view of a psychologist which is quite original. Piaget's theory is innovative in that he champions for an interdisciplinary approach, he poses new tracproblems and the use of strategies in the posing of the problems and the way to resolved them.

genetic psychology / epistemology / constructivism / Piaget

Correo electrónico: Silvia.Parrat-Dayan@unige.ch 
Piaget es, sin duda, uno de esos pensadores geniales que logra transformar profundamente concepciones mantenidas a lo largo de siglos, y consigue plantear problemas nuevos que otros no veían. Piaget intenta definir una nueva teoría del conocimiento.

El pensamiento piagetiano original y singular por su método de aproximación de los fenómenos, provocó reacciones contrarias, de adhesión o de rechazo. Asimismo, es precursor de novedades $\mathrm{y}$, además, de una actualidad que se resiste al paso del tiempo.

Podemos afirmar que el pensamiento piagetiano es una referencia obligada para el psicólogo del desarrollo.

Si tomamos uno a uno los conceptos y los problemas que interesan a los psicólogos del desarrollo cognitivo veríamos que todos surgieron en un marco piagetiano. Ningún psicólogo que se plantea los problemas cruciales del desarrollo (qué cambia, qué permanece, cómo ocurren los cambios, etcétera) ignora la obra de Jean Piaget, sea para criticarlo y oponerse a sus ideas, o para buscar nuevas interpretaciones de su pensamiento.

Treinta años después de su muerte, Piaget no puede ser considerado como una figura que ha pasado a la historia; renovó nuestra concepción del pensamiento del niño y supo desarrollar las pruebas experimentales que muestran que los modos del pensamiento infantil son diferentes que los del adulto (Bideaud et al., 1993).
No solo descubre nuevos problemas para la psicología, sino que se las ingenia para inventar situaciones muy sencillas en las que pone a prueba sus hipótesis. Debemos considerar que Piaget no contaba con ninguno de los instrumentos que facilitan el trabajo del científico, ni procesador de textos y programas estadísticos ni videos ni accesorios que permitan medir con exactitud el recorrido visual de los ojos del bebé, el grado de dilatación de la pupila, la actividad eléctrica de su cerebro, entre otros.

Piaget sorprende por su fuerza innovadora. Una característica fundamental de sus trabajos es la naturaleza interdisciplinaria. Su trayecto intelectual y científico estuvo siempre marcado por la integración de diferentes disciplinas. Un ejemplo es el Centro de Epistemología que creó y donde se rodeó de especialistas de diferentes disciplinas científicas, porque estaba convencido de que lo que cada uno podía aportar desde su especialidad era indispensable para un estudio completo del conocimiento. Destaquemos la actualidad de este enfoque interdisciplinario en el estudio de la cognición y de la psicología en general.

\section{LA CONTRIBUCIÓN A LA PSICOLOGIA}

La contribución de Piaget a la psicología es de primer orden. Piaget revolucionó la psicología. Su manera de proceder sigue una estrategia que consiste en definir dos posiciones opuestas y 
luego redefinir el campo entero, proponiendo una tercera solución (Bideaud et al., 1993).

Por ejemplo, uno de los problemas importantes que la psicología provocó desde el punto de vista epistemológico es el de la oposición, que aún hoy existe entre nativismo y ambientalismo en la definición de la cuestión herenciamedio. Aquí la posición de Piaget es conocida: se niega a elegir entre los dos factores y propone un modelo abstracto que los engloba y los trasciende, el de la equilibración. Esta estrategia es típica en Piaget: define dos posiciones opuestas vigentes y redefine la problemática señalando una tercera vía, un tercium.

La oposición innata adquirida no tiene sentido en la perspectiva de Piaget. No se trata de saber cuánto de innato y cuánto de adquirido interviene en la determinación de la conducta inteligente. Tampoco se trata de creer que todo está en el patrimonio genético o, al contrario, que todo está en la experiencia con el medio. El relativismo interaccionista, llamado más tarde constructivismo, se aparta tanto de la posición empirista como de la posición apriorista o innatista. Preguntarse qué proporción de innato y de adquirido determinan el comportamiento inteligente no es una pregunta piagetiana. Primero, porque la inteligencia no es una cantidad que se pueda medir sino una estructura, una organización. Por otro lado, porque lo que viene de la herencia y lo que vie- ne del medio no son componentes fijos que determinan el resultado del trabajo intelectual sin afectarse mutuamente y sin relación con las estructuras elaboradas por el sujeto. $\mathrm{Y}$, además, porque el medio en la teoría de Piaget no es un estímulo que provoca una respuesta, sino que es el propio niño, con su organización intelectual, quien va a buscar datos en el medio, datos que podrá asimilar o a los que podrá acomodarse. No hay una lectura pura de la experiencia. Lo importante radica en que es la conducta la que determina el medio. La iniciativa pertenece al niño.

Otro problema provocado por la psicología es el del paralelismo psicofísico. En realidad, en la perspectiva de Piaget este paralelismo es un problema falso, ya que existe un isomorfismo necesario entre los sistemas implicativos de significado a nivel consciente y el sistema causal a nivel material o fisiológico. "Material" y "significado" son dos caras de la misma moneda: las explicaciones causales están finalmente basadas en un sistema de implicaciones deductivas y las implicaciones deductivas están basadas en un sustrato material causalmente organizado. La profundización de la causalidad va junto con una mayor comprensión de la implicación porque la causalidad acaba en una atribución a la naturaleza de los poderes deductivos del sujeto.

Otra característica innovadora del pensamiento de Piaget que explica la riqueza y la fecundidad de sus obser- 
vaciones y que tiene relación con el hecho de que no hay una lectura pura de la experiencia, es la necesidad de tener una teoría explícita que guíe y dé sentido a las observaciones. De nuevo, Piaget se distingue de sus contemporáneos. Por eso, en vez de observar a los niños de manera naturalista, sin plantearse muchas preguntas y apoyándose en una epistemología ingenua e implícita, que consiste en pensar que un observador atento puede leer directamente en el comportamiento del niño, Piaget plantea de nuevo una posición revolucionaria. Porque Piaget no piensa que se pueda leer directamente el comportamiento del niño; al contrario, piensa que el observador construye su lectura. En esta construcción Piaget se sitúa entre el idealismo y el empirismo en filosofía. Si el idealismo prioriza las reglas internas con relación a los hechos externos, el empirismo va a privilegiar los hechos y no las normas. La perspectiva piagetiana, que es el constructivismo, postula que hechos y normas son siempre proporcionales. O sea que a cada profundización de los hechos corresponde una profundización de las normas, y viceversa.

La novedad más radical de la epistemología piagetiana reside en la búsqueda de una fundamentación empírica y científica de sus hipótesis, fundamentación que le da el método histórico crítico y sobre todo la psicología genética que Piaget elabora durante más de cuarenta años. Para
Piaget la reflexión es un punto de partida que le permite plantear una serie de problemas pero no una manera de resolverlos. La verificación y el control de estos problemas son necesarios para evitar la especulación (Meljac et al., 1998).

\section{El proyecto de Piaget}

Es a través de la psicología que las ideas de Piaget van a tener una gran influencia en el pensamiento tanto psicológico como pedagógico. Sin embargo, el proyecto de Piaget no era la psicología; no fue nunca el de comprender el niño como tal. El proyecto de Piaget fue de orden epistemológico. La epistemología es una teoría del conocimiento. Como los filósofos, Piaget se interesa en la teoría del conocimiento, específicamente en el conocimiento científico. Pero en vez de preguntarse como los filósofos, qué es el conocimiento o cómo el conocimiento es posible, Piaget se preguntará cómo se pasa de un estado de conocimiento menor a un estado de conocimiento superior en el sentido de más potente. ¿Cómo se potencia el conocimiento? ¿Cómo el hombre se volvió un sujeto que conoce? ¿Cuáles son los mecanismos que permiten la emergencia de "lo nuevo"? ¿Cómo es que la inteligencia del ser humano, que prolonga sobre el plano mental la adaptación biológica se desarrolla en la dirección de una conquista asimiladora del universo cada vez más acelerada? Piaget 
va a tratar estas preguntas como psicólogo y no como filósofo. Y esta es la originalidad de su programa. Si Piaget formula de otra manera las preguntas es porque está buscando las respuestas en la ciencia. Y, efectivamente, las preguntas están formuladas como preguntas para ser estudiadas científicamente, es decir con control experimental. Para dar respuesta a estas preguntas Piaget se propone estudiar la génesis de las funciones cognitivas en el niño. La interrogación epistemológica en Piaget es constitutiva de una psicología genética que estudia las estructuraciones lógicas de la inteligencia, de una postura que él llama constructivismo y que se apoya en la actividad del sujeto y de un método que el propio Piaget define como un método clínico.

La finalidad de Piaget será entonces elaborar una epistemología genética, una teoría del conocimiento científico fundamentada en el estudio de la génesis psicológica de nociones, de operaciones y de estructuras intelectuales. El núcleo principal de la problemática piagetiana será el estudio de la construcción del pensamiento racional. Piaget estudiará la génesis de la lógica en el niño. Precisemos que en la teoría de Piaget la palabra genética no se refiere a los mecanismos de la herencia como en biología. En la teoría de Piaget, genética quiere decir desarrollo individual. La génesis es el conjunto de hechos que contribuyen a la formación de algo, o mejor, es la manera según la cual algo se formó.

La psicología, digámoslo de nuevo, era para Piaget una manera de enfocar el problema, un método que le permitiría encontrar la solución de un problema que hasta entonces era un problema de filósofos. Y, para responder a estos problemas, o sea el origen del conocimiento científico y la función adaptativa del conocimiento, Piaget va a interrogar a los niños. Idea escandalosa, porque como afirma Emilia Ferreiro, ¿cómo tener la idea de interrogar a los niños para encontrar respuestas a problemas para los cuales la reflexión filosófica había dedicado tantos esfuerzos durante tantos años? La idea era escandalosa para los que pensaban que los niños no sabían nada o lo que sabían era gracias al adulto que se los enseñó.

Piaget dice lo contrario. Interrogando a los niños Piaget muestra que ellos tienen ideas y que construyen su mundo haciendo teorías sobre la realidad exactamente como los científicos construyen teorías con respecto a este mundo. El niño no es un sujeto pasivo que recibe influencias del medio y responde en función de estas influencias. No es una especie de adulto en miniatura cuya única tarea sería ensanchar o agrandar los órganos del conocimiento. Piaget nos habla de un niño que no solo responde a las estimulaciones del medio sino que las re-estructura. Y, si al principio existe una cierta estructuración, el 
niño va a crear nuevos instrumentos de conocimiento que no tenía al principio. ¿Cómo? A través de un continuo intercambio con el medio.

Según Piaget el conocimiento no está ni en el sujeto ni en el objeto. Es imposible disociar el objeto del sujeto porque el sujeto se define con relación al medio y el medio tiene sentido cuando es interpretado por el sujeto. Por eso, la posición de Piaget no es ni inneista ni empirista, sino interaccionista. Se opone así a los psicólogos behavioristas, que piensan que los conocimientos se inscriben, a través del aprendizaje, en el cerebro, que es considerado como una especie de cera blanda. Piaget se opone también a los gestaltistas, que consideran que el cerebro contiene estructuras innatas sobre las cuales se suman, se agregan los conocimientos.

Para Piaget los conocimientos no vienen ni de fuera ni de dentro sino que provienen de la interacción. Piaget no elige ni el inneismo ni el empirismo sino que reformula el problema estudiado proponiendo una nueva posición: el interaccionismo y el constructivismo. Los puntos esenciales de la psicología piagetiana, o sea la focalización en los hechos normativos que provienen de las estructuraciones lógicas de la inteligencia y el rol fundamental de la acción al interior de un constructivismo que reúne el sujeto y el objeto, provienen de una epistemología en donde la psicología genética se torna el terreno experi- mental. Y es en este punto que se sitúa la originaliad de la teoría operatoria de Piaget que es, en realidad, la teoría de la universalidad de los instrumentos racionales del conocimiento.

El niño no fue el objeto de estudio de Piaget sino lo que le permitió resolver problemas de orden epistemológico. Y por esto mismo dedicó más de cuarenta años a elaborar una psicología del niño.

Podríamos decir que lo que los niños le dieron a Piaget le sirvió para comprender la génesis de la razón humana, para ayudarnos luego a nosotros a comprender mejor a los niños. Más exactamente, nos ayudó a entender el desarrollo intelectual del niño. Es de esta relación de intercambio con el niño que proviene probablemente la actitud de respeto, y en particular de respeto intelectual, que tenía en relación con ellos.

Piaget considera al niño como un ser humano que tiene cosas que decirle y enseñarle. Por eso, siempre fue un oyente atento a todas las respuestas del niño, incluso las respuestas vacilantes, dudosas, inesperadas y las aparentemente tontas. Es que el niño que Piaget interroga, no es un receptáculo pasivo sino una fuente de conocimientos. Si nos parece que dice algo sin sentido, es porque lo estamos comparando con los códigos de la norma adulta. Pero por cierto que tiene un sentido, y precisamente Piaget quería comprender cuál era este, ya que nos revelaba el proceso 
de construcción de novedades. Escucha al niño con atención y con una escucha creadora.

Podemos decir que Piaget fue un investigador interesado en la emergencia de la novedad. Y, ¿cómo se produce lo nuevo, la novedad? La tarea que se propuso Piaget -constantemente renovada y enriquecida con nuevas ideas- fue la de comprender los mecanismos del progreso del conocimiento.

\section{Explicar el Misterio del deSARROLLO CONSTRUCTIVO}

Piaget tuvo siempre como finalidad tratar de comprender y de explicar qué es el desarrollo en su continua construcción de novedades y en su progresiva adaptación a la realidad. Como él mismo decía, quería explicar el misterio del desarrollo constructivo.

Entonces se plantea múltiples preguntas: ¿Cómo es que el ser humano pasa solo en algunos años, de un estado de dependencia total a un nivel de capacidad intelectual que le permite asimilar las más sofisticadas teorías científicas hechas por la colectividad humana? ¿De dónde proviene lo nuevo en el niño? ¿Cómo es que progresa en su razonamiento? ¿Por qué muestra un día tanta seguridad en la respuesta que da a un problema y con la misma seguridad, pero algunos meses después, responde de manera totalmente diferente? ¿Cómo aumenta, cómo se acrecienta el conocimiento? ¿Cuáles son las leyes y los mecanismos que regulan el acrecentamiento del conocimiento humano? ¿Cómo es que el ser humano se adapta a su medio?

A todas estas preguntas Piaget les dará una respuesta.

Los principios sobre los cuales se sustentan las respuestas de Piaget pueden resumirse en tres.

Según el primer principio, la representación que el individuo hace de la realidad depende de instrumentos lógicos. Estos instrumentos organizan lo que percibimos y lo que creamos mentalmente.

El segundo principio indica que estos instrumentos lógicos no están programados por la especie ni impuestos como tales por el medio, sino que son el fruto de una reconstrucción individual a partir de nuestra propia actividad.

El tercer principio indica que la construcción del conocimiento es el fruto de una reconstrucción continua a través del intercambio entre el sujeto y el medio humano o físico.

La inteligencia se construye y en la base de esta construcción está la acción. Es la forma más general de la coordinación de acciones interiorizadas, acciones interiorizadas y reversibles, dice Piaget.

O sea que la inteligencia es la forma más general de las operaciones del pensamiento. Definida de esta manera la inteligencia corresponde a una expe- 
riencia de tipo lógico entre el sujeto y la realidad. La inteligencia no es lo mismo que el lenguaje, lo cual no quiere decir que el lenguaje no sea necesario para la construcción de la inteligencia. Y, lo que va a ser la llave de la inteligencia es lo que Piaget llama reversibilidad. La reversibilidad designa las coordinaciones entre la acción directa y la que la anula, o sea la inversa, o entre la acción directa y la que la compensa, o sea la recíproca. Todas las operaciones del pensamiento son reversibles. La reversibilidad implica la posibilidad de volver al punto de partida. Por ejemplo, cuando se le presentan al niño dos bolas de plastilina (A y B), y una vez que el niño establece la igualdad entre ellas, se modifica una de ellas (se hace una salchicha, por ejemplo) y se le coloca la pregunta de conservación: ¿Y ahora? ¿Hay lo mismo en A y en B o hay más en A que en B? El niño que tiene la noción de conservación nos dirá que es lo mismo porque podemos volver a hacer la bola como antes. Aquí no se trata de un retorno empírico. Es el pensamiento que vuelve para atrás anticipando los resultados.

Existe también la reversibilidad por reciprocidad. La operación recíproca no es la inversa. Ella no anula el resultado de la acción directa, sino que reconoce el resultado como de dos acciones que se compensan mutuamente: por ejemplo, la salchicha es más larga pero más fina, las dos dimensiones se coordinan y se compensan una con la otra.
De esta forma, el pensamiento puede mirar desde arriba los fenómenos inmediatos, los resultados de las acciones, porque el pensamiento es libre de anularlos o compensarlos sin necesidad de tocarlos.

Ahora bien, se llega a la reversibilidad después de un largo trabajo cognitivo. El progreso cognitivo es el producto de la actividad del sujeto. El conocimiento se construye por la acción.

Debemos aun recordar una idea central: Piaget liga el problema de la adaptación biológica al problema del pensamiento. Para Piaget, el conocimiento prolonga la adaptación biológica. Según él, la adaptación de todo organismo vivo como la de toda conquista intelectual consiste en la asimilación de un hecho externo, y esta se entiende como una transformación en sí misma. Un conejo que come repollo, dice Piaget, no se transforma en repo1lo, sino que transforma el repollo en conejo. De la misma manera, el conocimiento no es una copia de la realidad sino la integración de la realidad en una estructura mental preexistente que será más o menos modificada por esta integración. Como el conocimiento está fundamentado en la actividad del sujeto, podemos decir que este no es estrictamente objetivo. El objeto, decía Piaget, es conocido a través de la acción del sujeto, por eso debe ser reconstruido y, a causa de ello, se torna un límite que se trata de alcanzar indefinidamente, pero nunca se alcanza. 
La afirmación de Piaget cuando dice: la inteligencia organiza el mundo y se organiza a sí misma, indica la interdependencia de la construcción de la inteligencia, que nos permite comprender el mundo y nuestra acción, que es la fuente de cambios en esta comprensión. De esta forma, la evolución del ser humano depende de un intercambio sin fin entre acción y construcción. Es dentro de esta confrontación renovada eternamente que se construyen los nuevos conocimientos y se estructuran los nuevos instrumentos intelectuales. Toda construcción llega a un nivel de organización, pero siempre es relativa porque siempre permanece activa. Toda construcción nueva incita a la acción y es causa de nuevas construcciones, siguiendo un proceso que no tiene fin (Piaget, 1971).

El desarrollo mental constituye una creación continua de novedades y se caracteriza por una serie de innovaciones, que además aparecen como si tuvieran una dirección. O sea que lo nuevo no se da en cualquier orden sino que las innovaciones van de lo simple a lo complejo. En la teoría de Piaget, las adquisiciones anteriores son necesarias para las actuales y cada nueva adquisición es posible a causa de que las anteriores se integran en las nuevas (Piaget \& García, 1987).

De manera general y más descriptiva, podemos decir que en el transcurso del desarrollo se observan en el niño modificaciones de la conducta, que no se interpretan en términos cuantitativos de acumulación de conocimientos. Piaget recorta el desarrollo intelectual en grandes períodos cuyo orden de sucesión es constante y en el cual cada etapa resulta de la precedente a través de un proceso integrativo.

En cada etapa el individuo se encuentra centrado en su subjetividad y el desarrollo consiste en descentrarse. En una primera etapa, que se da en el plano de la acción, el niño aprende a disociar su propio cuerpo del medio ambiente. En un segundo momento, ya en el plano de la representación, el niño debe descentrarse de su propio punto de vista, que es lo que caracteriza el egocentrismo, es decir la incapacidad de colocarse en el punto de vista del otro, llegando a relativizar su juicio sobre la realidad. La tercera descentración, propia del adulto, consiste en relativizar los ideales y los sistemas del mundo, que en la adolescencia se piensan como universales.

En el transcurso de estos diferentes niveles, el niño inventa los instrumentos de su propio conocimiento; es un creador. La fuerza de los instrumentos intelectuales que el niño construye asegura la aparición de novedades desde el punto de vista del conocimiento.

B. Inhelder, una de las colaboradoras más próximas de Piaget, dijo en una entrevista:

La característica más llamativa de Piaget parece ser su deseo constante de descubrir datos nuevos y ampliar, de esta forma, su visión del conocimiento 
[...]. Para él la alegría de descubrir fue un motor importante de su investigación (Bideaud et al., 1993).

Digamos incluso con Jacqueline Bideaud (Bideaud et al., 1993) que, a diferencia de otras teorías que proponen como modelo de la vida cognitiva el animal (behaviorismo), el campo electro-magnético (Gestalt) o la computadora (cognitivimo), en la teoría de Piaget el sujeto humano es actor y autor de los objetos de sus conocimientos. Las estructuras normativas del conocimiento que fundan a la vez la objetividad y la subjetividad, son las estructuras de operaciones de un sujeto. El modelo no es ni el animal ni el campo de fuerzas físicas ni la máquina que calcula. El niño experto en desarrollo y el experto científico se explican mutuamente. No se sale del sujeto. La inteligencia encuentra en ella misma su propia explicación.

Resumiendo, Piaget estudió la creación de novedades al mismo tiempo que inventaba y creaba. Por ello, además de crear una teoría poderosa del conocimiento, nos enseña que el ser humano no es una víctima pasiva ni de necesidades biológicas ni de las fluctuaciones aleatorias del medio. El sujeto es un inventor y también un inventor de su propio futuro. Piaget nos deja un mensaje optimista y lleno de esperanza.

\section{Trabajando con Piaget}

No olvidemos que este punto de vista sobre el desarrollo interroga la educa- ción. Es verdad que las ideas de Piaget han tenido una enorme repercusión en el mundo de la educación; sin embargo, las relaciones entre la teoría piagetiana y la pedagogía son confusas. En realidad, siempre se ha planteado mal el problema porque se pensaba que la teoría de Piaget era la llave para solucionar todos los problemas de aprendizaje en la escuela. Así, se pensó en proponer, por ejemplo, un currículo escolar en función de los temas estudiados por Piaget, o en la necesidad de inspirarse en los estadios del desarrollo para decidir en qué momento se debía enseñar tal o cual noción. Evidentemente, todo esto fue un fracaso.

Todo proyecto educativo que quiera inspirarse en Piaget no debería aplicar de manera mecánica los datos encontrados por el autor; al contrario, debería guiarse por los principios y no por los resultados en relación con las nociones.

\section{¿̇Y DESPUÉS?}

No podemos negar que existe una cierta desafección hacia la teoría de Piaget de parte de los psicólogos (Meljac et al., 1998), que han hecho una lectura mucho menos epistémológica de su obra. Se interesaron más en las observaciones hechas por Piaget y sus colaboradores en distintos dominios y en edades diferentes y se fascinaron por sus experiencias, que constituyen las pruebas más ingeniosas que la psicología del desarrollo inventó. Así fue que entre los años 1965 y 1980, numerosas investiga- 
ciones tuvieron como objeto confrontar o rechazar las interpretaciones piagetianas. Estas investigaciones fueron la mayoría de las veces muy ambiguas. En el caso de los psicólogos anglosajones, estos rechazaban el método clínico, el pedido de justificaciones y las hipótesis alternativas, porque utilizaban las pruebas piagetianas como si fueran test, mostrando así que no entendían cuál era el propósito de Piaget. Y de parte de los psicólogos piagetianos también había cierta ambigüedad, puesto que rechazaban que las pruebas no evaluaban solo una estructuración subyacente, sino que también evaluaban los conocimientos empíricos que resultan del intercambio del niño con el medio. Por eso, estas investigaciones suscitaron lo que podríamos llamar un lenguaje de sordos, y condujeron a intercambios poco productivos. Es más, esto llevó -incluso entre los propios psicólogos piagetianos- a un rechazo no merecido de las pruebas piagetianas y a una focalización exclusiva en el estudio del funcionamiento cognitivo, asociado a un cierto abandono del estudio de las mismas estructuraciones lógicas.

Pero las concepciones de base de la teoría de Piaget son fuertes y resurjen allí en donde uno no se lo esperaba, por ejemplo en el tema del número, en el cual los hechos normativos son evocados por un investigador cognitivista que no conoce Piaget, o en el tema de la imagen mental, cuando se redescubre el rol de la motricidad y el lugar de la acción en el desarrollo cognitivo del sujeto, hechos evocados por neurofisiólogos actuales, que tampoco conocen a Piaget.

Con respecto a su teoría, el mismo Piaget decía que el esqueleto general que descubrió es más o menos evidente, pero que está aún con muchas lagunas. Él pensaba que cubriendo estas lagunas se podrían diferenciar de múltiples maneras las articulaciones encontradas. Y esto sin contradecir las grandes líneas del sistema propuesto, pero integrándolas en nuevas proposiciones. Asimismo, afirmaba que cuando una teoría sucede a otra, la primera impresión es que la contradice o la elimina. En realidad, decía Piaget, las investigaciones que continúan realizándose conducen a retener más de lo que se había previsto, de tal forma que la mejor teoría es la que consigue integrar el máximo de elementos obtenidos de sistemas anteriores.

Hoy, la teoría de Piaget no tiene una teoría alternativa válida, o sea otra teoría que explique todo lo que la teoría piagetiana explicó y además otros fenómenos que no explicaba. Por eso, no es difícil imaginar que se puedan desarrollar aún los temas piagetianos.

Se podrían tratar e investigar diferentes problemas; por ejemplo, la necesidad de explicar la interacción entre aspectos internos y externos del conocimiento. Al respecto, Piaget prioriza los aspectos internos, de allí la importancia de plantear un estudio de la relación entre ambos mecanismos. Otro tema que podría desarrollarse es el de 
las relaciones entre mecanismos generales, que son los que estudió Piaget, y mecanismos específicos del desarrollo. Tanto las asincronías que aparecen en el desarrollo de conocimientos diferentes (social, matemático, físico, notacional, lingüístico) como el grado de especialización de las competencias tempranas del bebé en los mismos dominios, requiere este tipo de estudio. Las perspectivas actuales del desarrollo se enriquecerían al integrar más elementos de la teoría piagetiana.

Es probable que la futura teoría del desarrollo consiga articular los aspectos diferenciales y universales de la conducta humana. Más allá de la psicología, podemos decir, con Jacqueline Bideaud (Bideaud et al., 1993), que si la variabilidad interindividual puede enriquecernos, es la universalidad, y en parte los instrumentos racionales del conocimiento que fascinaron a Piaget y que son los mismos en todas las razas, tiempos y espacios, la que nos reunirá. De nuevo, Piaget nos deja un mensaje de esperanza.
Considerando que los aportes de las teorías actuales son insuficientes, y teniendo en cuenta las experiencias e hipótesis que suscitan, psicólogos, educadores e investigadores podrían encontrar en la postura piagetiana elementos de reflexión que ayuden a pensar, tal vez de manera más justa, los acontecimientos del desarrollo.

\section{Referencias}

Bideaud, J., Houdé, O., \& Pedinielli, J.-L. (1993). L'homme en développement. París: Presses Universitaires de France.

Meljac, C., Voyazopoulos, R., \& Hatwell, Y. (Comp.) (1998). Piaget après Piaget. Saint-Etienne: La Pensée Sauvage.

Piaget, J., \& García, R. (1987). Vers une logique des significations. Ginebra: Murionde.

Piaget, J. (con la colaboración de R. García) (1971). Les explications causales. París: Presses Universitaires de France. 